\title{
THE MICRO-PRECISION INTERFEROMETER TESTBED INSTRUMENT DESIGN
}

\author{
B. HINES, G. NEAT, M.M. COLAVITA, R. CALVET, and L. SWORD \\ Jet Propulsion Laboratory, 4800 Oak Grove Drive, \\ Pasadena, California, USA
}

\begin{abstract}
The Micro-Precision Interferometer Testbed is essentially a space-based Michelson interferometer suspended in a ground-based laboratory. The purpose of the testbed is to serve as a proving ground for technologies needed for future space-based missions requiring low-vibration environments. A layered control architecture, utilizing isolation, structural control, and active optical control technologies, allows the system to achieve its vibration attenuation goals.

This paper focuses primarily on the interferometer instrument design for the testbed and the systems-level tradeoffs between the instrument and other systems due to the fact that the interferometer is on a large, lightly damped, flexible structure rather than on the ground. The testbed is designed to be a fully functioning interferometer spacecraft and makes use of flight-like hardware where possible, including an external star simulator, an attitude control system, fringe detection and tracking systems, delay lines, pointing control, laser metrology systems, and computers and electronic subsystems. The engineering decisions that led to the current instrument configuration are presented and explained.
\end{abstract}

Key words: MPI -optical interferometry -interferometer testbed -space-based interferometry

\section{Purpose of the Testbed}

The Micro-Precision Interferometer (MPI) Testbed, essentially a space-based optical interferometer suspended in a ground-based laboratory, is intended to serve a dual function. It is intended to serve as a ground-based incarnation of a flight-ready longbaseline stellar optical interferometer, inasmuch as is practical, and as a vehicle for research into and development of interferometer and vibration control subsystems that are planned or proposed for future missions.

In its planned configuration, the testbed includes subsystems for both simulated stellar interferometric observations and vibration isolation and control. The Testbed is patterned after the JPL Focus Mission Interferometer ${ }^{1}$, which is related to proposed space-based interferometers such as $\mathrm{OSI}^{2}$ and POINTS. ${ }^{3}$

\section{Description of the Testbed}

\subsection{General Configuration}

The basic testbed platform is constructed from hollow aluminum tubes that form a trusswork that is joined together at nodes consisting of aluminum balls approximately $7 \mathrm{~cm}$ in diameter (see photo in Figure 1). Such a platform was chosen because of the ease with which its vibrational modes can be modeled using standard codes such as NASTRAN and because of its low mass.

The structure consists of three 5-m booms, one vertical boom that would house spacecraft subsystems such as reaction wheels, computers, communications, etc., a horizontal boom that is used to house the interferometer optics, and a boom canted $15^{\circ}$ up from horizontal that will be used to support a tetrahedral structure to which retroreflectors will be attached to provide reference points for laser metrology beams that will be used to monitor the positions of critical optical elements in 3 dimensions. 
A number of palettes for the mounting of optical elements are pseudo-kinematically attached to the top surface of the optics boom via supporting rods attached to the structure's node balls. These palettes are essentially standard optical breadboards that have been fabricated in special shapes. The mounting hardware supporting the palettes isolates them from structural vibrations above $\sim 100 \mathrm{~Hz}$, while the palettes themselves are rigid below $\sim 100 \mathrm{~Hz}$. The palettes provide a convenient and stable platform for optical assembly, allowing the use of standard optical mounts and hardware while at the same time having much less mass than a 5-meter long optical breadboard. Subdivision of optical hardware onto the various palettes was done with both optical and structural considerations in mind, so that elements that should not vibrate with respect to one another are located on the same palette.

A variable-length optical delay line and a fixed-length optical delay line are attached to the structure via pseudo-kinematic mounts. The fixed delay line is sufficiently rigid, and it already has specific mounting points for all its required components, so a palette is not needed. The moving delay line travels on a pair of rails. In space, any lack of rigidity in the rails is not important to first order since the delay line optics act as a cat's-eye retroreflector. However, under gravity load, the rails would sag enough to cause laser metrology errors as the delay line travels back and forth, so a trusswork resembling an old-fashioned train trestle is used to provide rigidity to the delay line rails to keep vertical deflections due to gravity to less than $0.5 \mathrm{~mm}$.

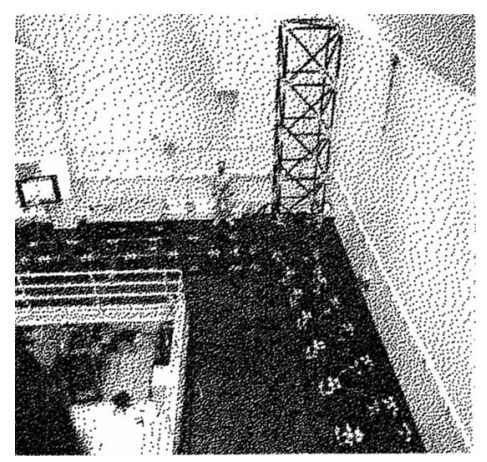

Figure 1. Photograph of the MPI Testbed.

\subsection{Optical Configuration}

The overall optical layout of the testbed in its initial configuration is shown in Figure 2. A description of the beam path through one arm of the interferometer is probably the most straightforward way to explain the system. The star simulator is distributed across a pair of optical tables on the laboratory floor next to the suspended structure. A laser (A) (or later a white-light source) on one table provides collimated light that is split into two beams that are then expanded to 4 inches in diameter. These beams then proceed to a pair of 6-inch siderostats (B) on the structure which are used to direct a 4inch clear-aperture beam into the interferometer. This beam is immediately compressed to 1 inch in diameter by a beam compressor (C). $90 \%$ of the beam then is reflected by a small piezoelectrically driven tip-tilt mirror (D) to keep the two incoming 
wavefronts jarallel. About $10 \%$ of the beam goes on to a wide-field-of-view camera (E) that is used for initial closed-loop acquisition of stars.

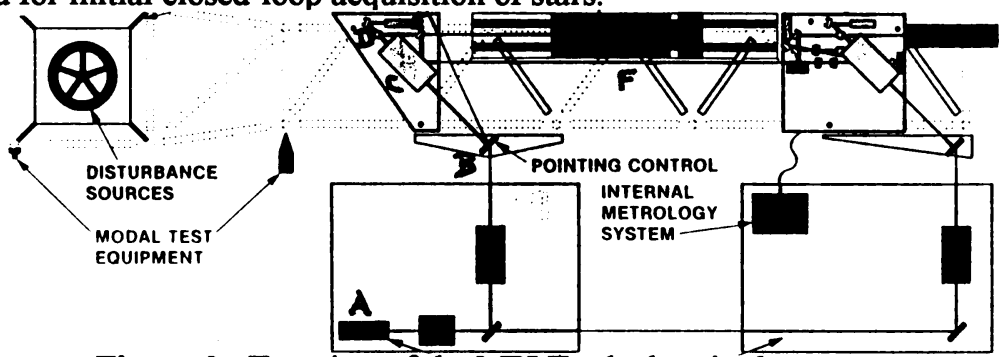

Figure 2. Top view of the MPI Testbed optics boom.

The light reflected from the tip-tilt mirror proceeds into the top aperture of the optical delay line $(\mathrm{F}) .^{4}$ The light then comes back out of the bottom aperture of the delay line, where it hits a pair of fold mirrors and proceeds on to the main beam combiner. On the beam combiner palette, the beam is reflected near-normal onto the main beamsplitter itself, where it interferes with the beam from the other arm of the interferometer. The central part of the combined beam then falls onto photon-counting detectors. The outer part of the beam is reflected into a fast-frame-rate CCD camera that is used as a sensor for wavefront tilt control. Due to an annular wedge in one arm of the interferometer, two separate star images are formed on the CCD when the wavefronts are parallel. ${ }^{5}$ Finally, infrared laser metrology beams monitor the pathlengths from the main beamsplitter back to the siderostats (internal metrology), and other metrology beams monitor the positions of the siderostats in three dimensions for use in astrometric observations (external metrology). ${ }^{2}$

\section{Current Status and Conclusions}

Although funding has been reduced from it original level, an initial setup involving an operating optical delay line controlling one beamsplitter-to-siderostat optical path is nearly complete. Also, a great deal of modal analysis data for the structure has been recorded and analyzed, ${ }^{6}$ and some effort has gone into the planning of vibration isolation and structural quieting efforts. ${ }^{7}$ Plans for FY94 call for the completion of the Testbed system as described in this paper.

\section{Bibliography}

[1] Laskin, R.A. and Martin, M.S., "Control/Structure system design of a spaceborne optical interferometer", AAS/AIAA Astrodynamics Specialist Conference, 1989.

[2] Gershman, R., Rayman, M.D., and Shao, M. "A moderate space mission for optical interferometry," 42nd Congress of the International Astronautical Federation, 1991, paper no. IAF-91-421.

[3] Reasenberg R.D. et al, "Microarcsecond optical astrometry, an instrument and its astrophysical applications," Astron. J. 96, No. 5, 1988, p. 1731 .

[4] Colavita, M., Hines, B.E., and Shao, M. "A high-speed optical delay line for stellar interferometry," ESO Conference on HighResolution Imaging by Interferometry II, 1991, Garching, Germany.

[5] Clark, L.D., "A photon-camera star tracker for stellar interferometry", SPIE Conference 627, Instrumentation in Astronomy VI, 1986, Tucson, Arizona, p. 838.

[6] Levine-West, M., Red-Horse, J., and Marek, E., "A systems approach to high fidelity modelling," 1st International Symposium on Microdynamics and Accurate Control, 1992, Nice, France.

[7] Spanos, J.T. et al, "Control structure interaction in long baseline space interferometers," 12th IFAC Symposium on Automatic Control in Aerospace, 1992, Ottobrunn, Germany. 